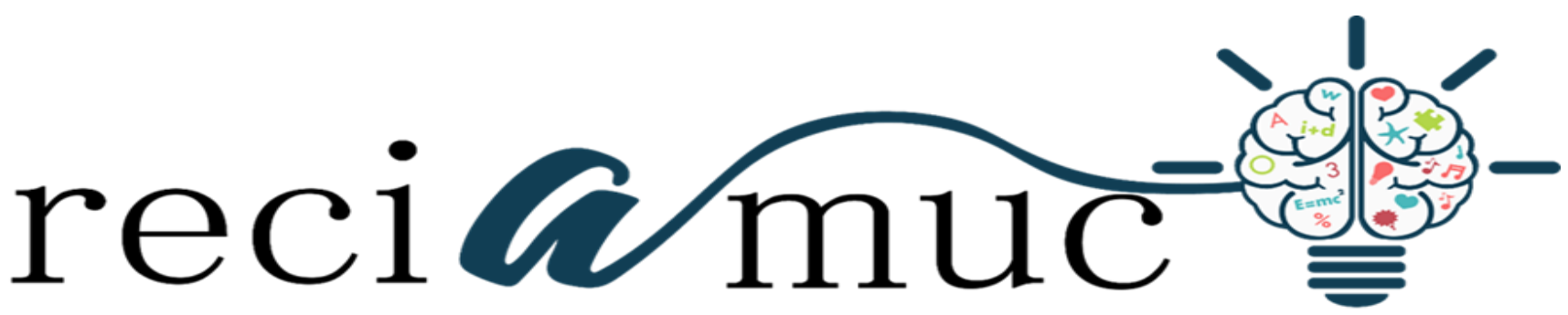

Revista cientifica de investigación actualización del mundo de las ciencias

José Gabriel Cayetano Lucas a; Mercedes María Lucas Choez b; Jaime Eduardo Jaramillo Soto ${ }^{\text {c; }}$ José Fernando Calderón Ponce ${ }^{\mathrm{d}}$; Román Andrés Ortiz Burgasi ${ }^{\text {e }}$ César David Pibaque Tigua ${ }^{\mathrm{f}}$

Protocolo de atención en pacientes sometidos a cirugía estética

Protocol of care in patients undergoing cosmetic surgery

Revista Científica de Investigación actualización del mundo de las Ciencias. Vol. 3 núm., 1, enero, ISSN: 2588-0748, 2018, pp. 847-866

DOI: $10.26820 / \mathrm{reciamuc/3.(1).enero.2019.847-866}$

URL: $\underline{\text { htp://reciamuc.com/index.php/RECIAMUC/article/view/261 }}$

Código UNESCO: 3205 Medicina Interna

Tipo de Investigación: Artículo de Revisión

Editorial Saberes del Conocimiento

Recibido: 10/12/2018

Aceptado: 20/01/2019

Publicado: 30/01/2019

Correspondencia: jogal91@hotmail.com
a. Médico Cirujano; jogal91@ @otmail.com; Quevedo, Ecuador.
b. Licenciada en Enfermería; mercedeslucas_ch86@ hotmail.com; Jipijapa, Ecuador.
c. Medico; jjaramillo666@hotmail.com; Santo Domingo, Ecuador
d. Médico Cirujano; josecalderon1993@hotmail.com; Guayaquil, Ecuador.
e. Médico; raob88@hotmail.com; Guayaquil, Ecuador.
f. Médico Cirujano; cesar_dav412@ hotmail.com; Jipijapa, Ecuador. 


\section{Protocolo de atención en pacientes sometidos a cirugía estética}

Vol. 3, núm. 1., (2019)

José Gabriel Cayetano Lucas; Mercedes María Lucas Choez; Jaime Eduardo Jaramillo Soto; José

Fernando Calderón Ponce; Román Andrés Ortiz Burgasi; César David Pibaque Tigua

\section{RESUMEN}

El mantenimiento de la seguridad del paciente en el quirófano es una de las principales preocupaciones de los cirujanos, hospitales y centros quirúrgicos. Eludir complicaciones prevenibles es esencial, y la presión para evitar estas complicaciones en la cirugía cos-metica está aumentando. Tradicionalmente, el personal de enfermería y anestesia han logrado cuestiones de posicionamiento y seguridad del paciente en la sala de operaciones. A medida que el número de procedimientos basados en oficinas en prácticas de cirugía estética van en incremento, comprender y aplicar las directrices de seguridad del paciente por el cirujano plástico tiene una importancia creciente. Se ha hecho una revisión de los aspectos más destacados del protocolo universal y requerimientos establecidos para prevenir las complicaciones perioperatorias. En el presente trabajo, se revisa la importancia de la aplicación de estas directrices en la práctica de cirugía cosmética. Los aspectos clave de la seguridad del paciente en la sala de operaciones se describen, incluyendo el posicionamiento del paciente, la protección ocular y otras cuestiones esenciales para la minimización de la morbilidad postoperatoria. Además, como la demanda de la cirugía de contorno corporal en la práctica cosmética sigue aumentando, prestando especial atención a las consideraciones de seguridad específicas para los pacientes obesos de pérdida de peso masiva.

Después de la revisión del presente documento, el lector debe ser capaz de introducir protocolo universal de la Comisión Conjunta en su práctica diaria. El lector comprenderá aspectos clave de posicionamiento del paciente, administración de la vía aérea y protección ocular en la cirugía estética. Por último, el lector tendrá una mejor comprensión de la asistencia perioperatoria de las poblaciones únicas, incluyendo los pacientes, pérdida masiva de peso con obesidad mórbida y los ancianos. La atención al detalle en estos aspectos de la seguridad del paciente puede ayudar a evitar complicaciones innecesarias y mejorar significativamente la experiencia del paciente y los resultados quirúrgicos.

Palabras Claves: Cirugía del contorno corporal, Cirugía cosmética, Seguridad del paciente. 


\title{
Protocolo de atención en pacientes sometidos a cirugía estética
}

Vol. 3, núm. 1., (2019)

José Gabriel Cayetano Lucas; Mercedes María Lucas Choez; Jaime Eduardo Jaramillo Soto; José Fernando Calderón Ponce; Román Andrés Ortiz Burgasi; César David Pibaque Tigua

\begin{abstract}
The maintenance of patient safety in the operating room is one of the main concerns of surgeons, hospitals and surgical centers. Avoiding preventable complications is essential, and the pressure to avoid these complications in cosmetic surgery is increasing. Traditionally, nursing and anesthesia personnel have achieved positioning and patient safety issues in the operating room. As the number of office-based procedures in cosmetic surgery practices increases, understanding and applying patient safety guidelines by the plastic surgeon is of increasing importance. A review of the most important aspects of the universal protocol and established requirements to prevent perioperative complications has been made. In the present work, the importance of the application of these guidelines in the practice of cosmetic surgery is reviewed. The key aspects of patient safety in the operating room are described, including patient positioning, eye protection and other essential issues for the minimization of postoperative morbidity. In addition, as the demand for cosmetic contour surgery in cosmetic practice continues to increase, paying special attention to specific safety considerations for obese patients of massive weight loss.
\end{abstract}

After reviewing this document, the reader should be able to introduce universal protocol of the Joint Commission in their daily practice. The reader will understand key aspects of patient positioning, administration of the airway and eye protection in cosmetic surgery. Finally, the reader will have a better understanding of the perioperative care of unique populations, including patients, massive weight loss with morbid obesity and the elderly. Attention to detail in these aspects of patient safety can help avoid unnecessary complications and significantly improve patient experience and surgical outcomes.

Key Words: Body Contouring Surgery, Cosmetic Surgery, Patient Safety. 


\section{Protocolo de atención en pacientes sometidos a cirugía estética}

Vol. 3, núm. 1., (2019)

José Gabriel Cayetano Lucas; Mercedes María Lucas Choez; Jaime Eduardo Jaramillo Soto; José

Fernando Calderón Ponce; Román Andrés Ortiz Burgasi; César David Pibaque Tigua

\section{Introducción.}

La demanda de procedimientos cosméticos esta cada día en aumento. Los cirujanos están encargados de mantener los más altos estándares de seguridad del paciente. En los últimos años, el tema de la seguridad del paciente en el cuidado de la salud se ha convertido en una de las principales preocupaciones del público. El artículo emblemático del Instituto de Medicina, To Err is Human, comparte la asombrosa estadística de que entre 44,000 y 98,000 de estadounidenses mueren cada año debido a un error médico (Kohn, Corrigan, \& Donnaldson, 1999). En este informe, los autores destacan la seguridad del paciente como un área clave en necesidad de mejora en los hospitales de hoy. La seguridad del paciente en las salas de cirugía es una gran preocupación para los médicos, hospitales y centros quirúrgicos, así como el funcionamiento óptimo de las instalaciones por igual. Los médicos tienen una inversión obvia en la seguridad de sus pacientes, y los cirujanos son los responsables en última instancia de toda la actividad que se produce durante un procedimiento quirúrgico.

Los hospitales y las instalaciones quirúrgicas son revisadas por muchas organizaciones que revisan específicamente los problemas de seguridad del paciente para determinar el estado de acreditación. A medida que la tendencia de cuidado para el paciente cosmético se traslada al servicio ambulatorio, manteniendo las directrices estrictas llevadas a cabo por las diversas acreditaciones, el grupo se convierte en responsabilidad del cirujano. Además, la crisis de mala praxis sólo aumenta el nivel de atención puesta en temas de seguridad en quirófano.

Debido a la naturaleza electiva de la cirugía estética, mantener la seguridad del paciente durante estos procedimientos es especialmente importante. En el ámbito hospitalario, gran parte del posicionamiento y preparación del paciente es completada por enfermeras y personal de anestesiología. Ahora que los cirujanos plásticos están realizando más procedimientos cosméticos en el entorno quirúrgico del consultorio, la importancia de entender las pautas de seguridad adecuadas y preoperatorias en la gestión del posicionamiento del paciente se convierte en un importante problema. 


\section{Protocolo de atención en pacientes sometidos a cirugía estética}

Vol. 3, núm. 1., (2019)

José Gabriel Cayetano Lucas; Mercedes María Lucas Choez; Jaime Eduardo Jaramillo Soto; José Fernando Calderón Ponce; Román Andrés Ortiz Burgasi; César David Pibaque Tigua

Las directrices recientes establecidas por la Comisión Conjunta, incluido el Protocolo Universal (Commission., 2009), exige la adhesión a ciertos estándares en la atención quirúrgica en caso de que el hospital permanezca con una instalación acreditada. En el presente artículo, varios aspectos claves de la seguridad del paciente en los quirófanos se resaltan. Una revisión de la articulación del Protocolo Universal de la Comisión explica los requisitos peri operatorios para instalaciones hospitalarias acreditadas y ambulatorias. También se revisan las consideraciones básicas de seguridad, incluyendo atención a la preparación preoperatoria, posicionamiento del paciente, protección corneal y manejo de la vía aérea. Cuestiones específicas para poblaciones únicas como las personas mayores y obesas, se discuten los pacientes con pérdida de peso masiva. La atención a estos problemas preoperatorios puede prevenir complicaciones que pueden convertirse en problemas significativos en el postoperatorio.

\section{Metodología.}

Para el desarrollo de este proceso investigativo, se plantea como metodología la encaminada hacia una orientación científica particular que se encuentra determinada por la necesidad de indagar en forma precisa y coherente una situación, en tal sentido (Davila, 2015) define la metodología "como aquellos pasos previos que son seleccionados por el investigador para lograr resultados favorables que le ayuden a plantear nuevas ideas".(p.66)

Lo citado por el autor, lleva a entender que el desarrollo de la acción investigativa busca simplemente coordinar acciones enmarcadas en una revisión bibliográfica con el fin de complementar ideas previas relacionadas al protocolo de atención en pacientes sometidos a cirugía estética a través de una revisión de literatura, para así finalmente elaborar un cuerpo de consideraciones generales que ayuden a ampliar el interés propuesto.

\section{Tipo de Investigación.}

Dentro de toda práctica investigativa, se precisan acciones de carácter metodológico mediante las cuales, se logra conocer y proyectar los eventos posibles que la determinan, así como las características que hacen del acto científico un proceso interactivo ajustado a una realidad 


\section{Protocolo de atención en pacientes sometidos a cirugía estética}

Vol. 3, núm. 1., (2019)

José Gabriel Cayetano Lucas; Mercedes María Lucas Choez; Jaime Eduardo Jaramillo Soto; José Fernando Calderón Ponce; Román Andrés Ortiz Burgasi; César David Pibaque Tigua

posible de ser interpretada. En este sentido, se puede decir, que la presente investigación corresponde al tipo documental, definido por Castro (2016), "se ocupa del estudio de problemas planteados a nivel teórico, la información requerida para abordarlos se encuentra básicamente en materiales impresos, audiovisuales y /o electrónicos”. (p.41).

En consideración a esta definición, la orientación metodológica permitió la oportunidad de cumplir con una serie de actividades inherentes a la revisión y lectura de diversos documentos donde se encontraron ideas explicitas relacionadas con los tópicos encargados de identificar a cada característica insertada en el estudio. Por lo tanto, se realizaron continuas interpretaciones con el claro propósito de revisar aquellas apreciaciones o investigaciones propuestas por diferentes investigadores relacionadas con el tema de interés, para luego dar la respectiva argumentación a los planteamientos, en función a las necesidades encontradas en la indagación.

\section{Fuentes Documentales.}

$\mathrm{El}$ análisis correspondiente a las características que predomina en el tema seleccionado, llevan a incluir diferentes fuentes documentales encargadas de darle el respectivo apoyo y en ese sentido cumplir con la valoración de los hechos a fin de generar nuevos criterios que sirven de referencia a otros procesos investigativos. Para (CASTRO, 2016) las fuentes documentales incorporadas en la investigación documental o bibliográfica, "representa la suma de materiales sistemáticos que son revisados en forma rigurosa y profunda para llegar a un análisis del fenómeno"'(p.41). Por lo tanto, se procedió a cumplir con la realización de una lectura previa determinada para encontrar aquellos aspectos estrechamente vinculados con el tema, con el fin de explicar mediante un desarrollo las respectivas apreciaciones generales de importancia.

\section{Técnicas para la Recolección de la Información.}

La conducción de la investigación para ser realizada en función a las particularidades que determinan a los estudios documentales, tiene como fin el desarrollo de un conjunto de acciones encargadas de llevar a la selección de técnicas estrechamente vinculadas con las características del estudio. En tal sentido, (Bolívar, 2015), refiere, que es "una técnica particular para aportar ayuda a los procedimientos de selección de las ideas primarias y secundarias”. (p. 71). 


\section{Protocolo de atención en pacientes sometidos a cirugía estética}

Vol. 3, núm. 1., (2019)

José Gabriel Cayetano Lucas; Mercedes María Lucas Choez; Jaime Eduardo Jaramillo Soto; José Fernando Calderón Ponce; Román Andrés Ortiz Burgasi; César David Pibaque Tigua

Por ello, se procedió a la utilización del subrayado, resúmenes, fichaje, como parte básica para la revisión y selección de los documentos que presentan el contenido teórico. Es decir, que mediante la aplicación de estas técnicas se pudo llegar a recoger informaciones en cuanto a la revisión bibliográfica de los diversos elementos encargados de orientar el proceso de investigación. Tal como lo expresa, (Bolívar, 2015) "las técnicas documentales proporcionan las herramientas esenciales y determinantes para responder a los objetivos formulados y llegar a resultados efectivos" (p. 58). Es decir, para responder con eficiencia a las necesidades investigativas, se introdujeron como técnica de recolección el método inductivo, que hizo posible llevar a cabo una valoración de los hechos de forma particular para llegar a la explicación desde una visión general.

Asimismo, se emplearon las técnicas de análisis de información para la realización de la investigación que fue ejecutada bajo la dinámica de aplicar diversos elementos encargados de determinar el camino a recorrer por el estudio, según, (Bolívar, 2015) las técnicas de procesamiento de datos en los estudios documentales "son las encargadas de ofrecer al investigador la visión o pasos que debe cumplir durante su ejercicio, cada una de ellas debe estar en correspondencia con el nivel a emplear" (p. 123). Esto indica, que para llevar a cabo el procesamiento de los datos obtenidos una vez aplicado las técnicas seleccionadas, tales como: fichas de resumen, textual, registros descriptivos entre otros, los mismos se deben ajustar al nivel que ha sido seleccionado.

\section{Resultados.}

\section{Los 'Time Out'.}

En 2003, la Sociedad Americana de Cirujanos Plásticos se unió a otras 50 asociaciones médicas importantes que suscriben protocolo universal de la Comisión Conjunta (Commission., 2009) para prevenir sitio equivocado, procedimiento equivocado y cirugía en persona equivocada. A partir del 1 de julio de 2004 todos los hospitales acreditados, la atención ambulatoria y las instalaciones de cirugía basados en oficina a integrar varios elementos incluidos la identificación del paciente preoperatorio, sitio de la operación de marcado y completar un 'tiempo de espera' 


\section{Protocolo de atención en pacientes sometidos a cirugía estética}

Vol. 3, núm. 1., (2019)

José Gabriel Cayetano Lucas; Mercedes María Lucas Choez; Jaime Eduardo Jaramillo Soto; José Fernando Calderón Ponce; Román Andrés Ortiz Burgasi; César David Pibaque Tigua

antes de hacer la primera incisión. Este mandato se produce después de varios eventos centinela destacaron los fallos de la seguridad del paciente con el sitio equivocado y la cirugía incorrecta del paciente.

El propósito del proceso de verificación preoperatorio establecido por el Protocolo Universal es para asegurar que los documentos pertinentes y los estudios necesarios son completos y precisos antes de iniciar un procedimiento. Para la cirugía cosmética esto a menudo incluye una historia completa y consentimiento físico, información exacta y actualizada y los documentos para su aprobación por los profesionales médicos, si es necesario. El Protocolo también pone de relieve la importancia de asegurar la presencia de todo el material implantable necesario para el procedimiento.

Debido a un número sorprendentemente alto de sitio incorrecto y cirugías, incluso erróneas del paciente, la Comisión Conjunta requiere ahora marcado del lugar de la operación en el área de espera preoperatoria. Marcando el paciente identifica de forma inequívoca la ubicación del procedimiento. Para el cirujano plástico, esto a menudo se completó justo antes del procedimiento en el marcado de la paciente de rutina. En muchos casos, como ritidectomía, esto parece innecesario y obvio. Para otros procedimientos, como la liposucción de las áreas múltiples, esto sirve como una oportunidad para revisar con el paciente y el cirujano las áreas particulares que deben abordarse.

El último componente del Protocolo Universal, el 'tiempo de espera', actúa como una verificación final del paciente correcto, el procedimiento y el sitio de la operación. Por otra parte, el cirujano aprovecha esta oportunidad para confirmar que todas las herramientas necesarias y los implantes están presentes y disponibles para la operación. El tiempo de espera se puede completar antes o después de la inducción, y debe incluir todo el equipo operativo. Las directrices explican que la 'activa comunicación' entre el cirujano, personal de anestesia y enfermera es necesario. Las directrices específicas no identifican que en realidad debe anunciar el tiempo de espera; sin embargo, no debe haber consenso entre todos los miembros participantes antes del inicio del procedimiento. 


\section{Protocolo de atención en pacientes sometidos a cirugía estética}

Vol. 3, núm. 1., (2019)

José Gabriel Cayetano Lucas; Mercedes María Lucas Choez; Jaime Eduardo Jaramillo Soto; José Fernando Calderón Ponce; Román Andrés Ortiz Burgasi; César David Pibaque Tigua

El número de procedimientos que se realizan en las instalaciones quirúrgicas fuera del ámbito hospitalario sigue aumentando. Además, el número de cirujanos plásticos que utilizan servicios de cirugía y cirugía en el consultorio está aumentando. La optimización de la seguridad del paciente en todas las instalaciones es de suma importancia para la mejor atención de los pacientes. Por esta razón, la Comisión Conjunta creó un Programa de Acreditación de Atención Ambulatoria para certificar estas instalaciones no basados en los hospitales. Incluido en los requisitos para la certificación es el cumplimiento estricto de las anteriores directrices del protocolo universal. Por estas razones, se hace cada vez más importante que los cirujanos plásticos entren en conciencia y apliquen estas directrices en sus prácticas cotidianas.

\section{La colocación del paciente.}

Posicionar al paciente antes del inicio de una operación es un elemento fundamental para el éxito del procedimiento. Un pobre posicionamiento del paciente puede dar lugar a un procedimiento más difícil y puede producir resultados menos satisfactorios. Además de facilitar la terminación lisa del procedimiento, la posición adecuada del paciente también puede prevenir múltiples potenciales morbilidades postoperatorias. Es imprescindible que tanto el anestesiólogo y el cirujano están de acuerdo en la posición antes de la operación para asegurar la protección de las vías respiratorias. El equipo también debe tener en cuenta que los pacientes anestesiados no pueden hacer al cirujano consciente de las posiciones comprometidas. Además, las comorbilidades médicas del paciente y sus limitaciones de posicionamiento deben ser considerados.

Los pacientes que se someten a un procedimiento que requiere un tiempo operatorio largo están en riesgo de necrosis por presión de la piel y el tejido subyacente. Los estudios han demostrado que una presión de sólo $70 \mathrm{~mm}$ de $\mathrm{Hg}$ aplicadas durante un periodo de $2 \mathrm{~h}$ o más puede resultar en isquemia tisular irreversible (Vandam, 1993). Áreas especialmente susceptibles incluyen la piel sobre la frente, crestas ilíacas y óseas en los brazos y piernas. Espumas o esponjas de espuma similares sobre estas áreas y sobre los talones en el paciente en posición supina pueden ayudar a disminuir el riesgo de lesiones. Además, la elevación periódica de la zona durante el procedimiento permite una mayor cantidad de flujo sanguíneo. 


\section{Protocolo de atención en pacientes sometidos a cirugía estética}

Vol. 3, núm. 1., (2019)

José Gabriel Cayetano Lucas; Mercedes María Lucas Choez; Jaime Eduardo Jaramillo Soto; José Fernando Calderón Ponce; Román Andrés Ortiz Burgasi; César David Pibaque Tigua

La lesión nerviosa periférica es por lo general una complicación prevenible de la mala colocación del paciente. Los nervios son lesionados por uno de dos mecanismos: estiramiento o compresión (Vandam, 1993). Si el nervio se tira entre dos puntos fijos, lesión por estiramiento se produce. La lesión de compresión se debe principalmente a la pérdida de tono muscular protector y la presión entre dos puntos fijos. El resultado final es el mismo: la isquemia del nervio debido al pobre flujo sanguíneo (Cucchiara \& Faust, 1994). Mientras que los pacientes que no están sedados sienten los efectos de la isquemia del nervio y es posible ajustar la posición del cuerpo, el paciente anestesiado es incapaz de sentir o responder a tales signos.

Hay tres grados de lesión del nervio. La primera es neuro-praxias, lo que lleva a un mejor pronóstico. Neuropraxia es una respuesta a la compresión con sólo una ligera evidencia de desmielinización y no degeneración axonal. Se espera una recuperación completa dentro de seis semanas sin pérdida de función residual (Cucchiara \& Faust, 1994). El segundo nivel de la lesión del nervio es axonotmesis, que se produce cuando hay destrucción de los axones dentro de una vaina del nervio que se mantiene intacta (Vandam, 1993). Los axones distales degeneran y los axones proximales utilizan la vaina del nervio intacto para guiar la regeneración a una velocidad de $1 \mathrm{~mm}$ por día. La función se pierde al principio, pero puede volver durante el período de meses a un año, dependiendo de la longitud del nervio. La lesión del nervio final y más grave es neuro tmesis, que resulta en la interrupción del axón, la vaina y la cubierta del tejido conectivo. La degeneración del axón distal se produce, pero es menos probable la recuperación del nervio y la regeneración (Cucchiara \& Faust, 1994). Dolorosos neuromas pueden desarrollarse debido a la regeneración axonal desorganizada. La intervención quirúrgica con resección de terminaciones nerviosas y reaproximación puede ayudar a recuperar la función.

En un estudio de la American Society of Anesthesiologists, las cuentas de la lesión del nervio para $15 \%$ de las reclamaciones legales, y los nervios más comúnmente lesionado incluyen el plexo braquial, nervio cubital y el nervio radial (Fritzlen, Kremer, \& Biddle, 2003). Estas lesiones generalmente no son permanentes, sino que incluso la pérdida temporal del plexo braquial es un resultado inaceptable para algunos pacientes después de un procedimiento cosmético electivo. 


\section{Protocolo de atención en pacientes sometidos a cirugía estética}

Vol. 3, núm. 1., (2019)

José Gabriel Cayetano Lucas; Mercedes María Lucas Choez; Jaime Eduardo Jaramillo Soto; José Fernando Calderón Ponce; Román Andrés Ortiz Burgasi; César David Pibaque Tigua

El plexo braquial y sus ramas.

La lesión del plexo braquial es una complicación bien documentada de procedimientos largos cuando los brazos están en ángulo recto con el cuerpo o en hiperextensión. (Akinbingol, Borman, \& Maral, 2002) reporto un caso de una mujer obesa que se sometió a mamoplastia de reducción bilateral, abdominoplastia y liposucción con denervación postoperatoria del plexo braquial bilateral. El paciente recuperó lentamente funciones motoras y sensoriales durante varios meses. La lesión del plexo braquial se debe a estirar entre el proceso coracoides y la cabeza del húmero. Este tramo se ve en 90 grados de abducción del brazo y se agrava cuando el brazo se hace más hiperextendido (Vandam, 1993). Los cirujanos plásticos deben ser conscientes de esta complicación, ya que es bastante común en la cirugía de mama para mantener los brazos del paciente en la posición de abducción. Los cirujanos deben preocuparse especialmente en pacientes obesos debido a que la tensión aplicada a los plexos puede ser mayor en un menor grado de abducción debido a la gran circunferencia del brazo (Akinbingol, Borman, \& Maral, 2002). En estos pacientes, el secuestro se debe limitar a 80 grados o menos para evitar lesiones. La flexión de la cabeza con desplazamiento hacia abajo del hombro también puede poner tensión indebida en el plexo braquial y debe evitarse.

Los nervios cubital y radial son ramas del plexo braquial que puede ser lesionado si es posicionado inapropiadamente. El síndrome de compresión del túnel cubital se produce porque el nervio cubital se encuentra superficial en el túnel en el codo. Algunos han postulado que el síndrome se produce cuando el brazo se fija en el lado del paciente o secuestrada con el antebrazo en pronación. En estas posiciones, el túnel cubital se coloca en contacto con la superficie de la mesa y está sujeta a la lesión por compresión. La supinación permite que el proceso olécranon para proteger el nervio de contacto con la superficie plana, posiblemente reduciendo el riesgo de lesiones. Además, la flexión del codo más de 90 grados provoca una disminución en el tamaño del túnel cubital en más de un $90 \%$.

Al igual que el nervio cubital, el nervio radial se encuentra superficial ya que se envuelve alrededor del tercio proximal del húmero en la ranura radial. La parálisis de este nervio es menos común que el cubital, pero el relleno inadecuado del brazo largo puede predisponer a una lesión 


\section{Protocolo de atención en pacientes sometidos a cirugía estética}

Vol. 3, núm. 1., (2019)

José Gabriel Cayetano Lucas; Mercedes María Lucas Choez; Jaime Eduardo Jaramillo Soto; José

Fernando Calderón Ponce; Román Andrés Ortiz Burgasi; César David Pibaque Tigua

de compresión y pérdida funcional temporal. Las recomendaciones actuales sugieren la protección de esta zona del brazo con relleno blando y evitar la cinta circunferencial en la zona del húmero proximal. Los estudios han demostrado, sin embargo, que incluso con el posicionamiento y el relleno apropiado algunos pacientes todavía sufren de parálisis del nervio.

\section{Posiciones quirúrgicas.}

Hay un amplio espectro de posiciones utilizadas en el quirófano. En la cirugía estética, sin embargo, las posiciones más comúnmente utilizadas incluyen, decúbito lateral, propensos, supino y sentado. Supino es la posición más común y permite al cirujano un acceso adecuado a los aspectos de la cara, pecho, abdomen y anteriores de los brazos, las piernas y los muslos. Dado que no hay gradiente de presión por encima o por debajo del corazón, las consecuencias fisiológicas sobre el sistema cardiovascular son mínimas. En la posición supina el cirujano debe estar al tanto de la cabeza y la columna cervical y asegurar que éstos son la línea media. Movimientos repentinos de la cabeza puede ejercer una tensión excesiva en el plexo braquial que causan lesión por estiramiento. Incluso hay informes de disección de la arteria vertebral después de movimiento vigoroso de la cabeza y el cuello durante la cirugía (Bund, Heine, \& Jaeger, 2005).

El posicionamiento apropiado de los brazos durante la cirugía de mama es importante para prevenir neuropraxia y para la creación de simetría de mama cuando la inspección de la paciente en la posición sentada durante el procedimiento. Un factor de complicación común es notar que los hombros del paciente no están al mismo nivel cuando el paciente está sentado para la inspección de simetría durante la operación. Esto conduce a la dificultad en la técnica operatoria y complica el logro de simetría. Es común mover al paciente de la posición supina a la posición de asiento varias veces durante un procedimiento de mama cosmético. Si los brazos no están asegurados adecuadamente, se deslizan fuera de las juntas del brazo que conducen a neuropraxia si no se corrige por el personal de anestesia en el momento oportuno. Con los brazos secuestrados, deben estar en un ángulo inferior a 90 grados con los codos ligeramente flexionados y las manos en la posición supina. En muchas instituciones, que comúnmente usan cuñas de espuma antes de que las almohadillas de caja de huevo, los brazos se fijan entonces a 


\section{Protocolo de atención en pacientes sometidos a cirugía estética}

Vol. 3, núm. 1., (2019)

José Gabriel Cayetano Lucas; Mercedes María Lucas Choez; Jaime Eduardo Jaramillo Soto; José Fernando Calderón Ponce; Román Andrés Ortiz Burgasi; César David Pibaque Tigua

esta base utilizando rollo de banda de algodón de una manera circunferencial suelto. Esto ofrece la mayor protección frente a la parálisis de las ramas del plexo braquial (Bund, Heine, \& Jaeger, 2005).

Los cirujanos plásticos utilizan la posición prona con más frecuencia que nunca. En la cirugía reconstructiva que ofrece acceso obvio para el sacro de las úlceras por presión, así como a los glúteos para su uso en los esfuerzos de reconstrucción. En la cirugía estética esta posición es útil para la liposucción, ascensores de cuerpo circunferenciales, ascensores del muslo y de la cirugía de la nalga. La posición prona normalmente se lleva a cabo por intubación y la protección de la vía aérea en la posición supina seguido de transición a la posición prona. Hay que tener cuidado para evitar la presión excesiva en los ojos, oídos, nariz, senos y genitales masculinos. Los requisitos básicos de relleno incluyen el uso de rollos de gel paralelos para elevar el pecho fuera de la mesa operatoria. Los rollos de gel pueden ser colocados en paralelo a la cama, lo que permite la colocación medial de los senos y genitales (Figura 1). Otros cirujanos, sin embargo, prefieren los rodillos de gel para ser colocado debajo del pecho y debajo del pubis. Esta búsqueda ha encontrado ningún dato para apoyar un método de elevación del pecho sobre otro. El objetivo sigue siendo, para dejar espacio para los pechos, el abdomen y los genitales para descansar libres de presión. Las almohadas o cajas de huevos de espuma deben ser colocados debajo de las rodillas y los tobillos para evitar la presión sobre estas prominencias óseas.

Figura 1. Mesa operativa para posicionamiento prono. La cara del paciente está protegida con una almohada prona y el pecho elevado con rollos de gel, lo que permite el desplazamiento medial de los senos y los genitales masculinos. Todas las áreas de prominencia ósea se deben rellenar con almohadas o cajas de espuma.

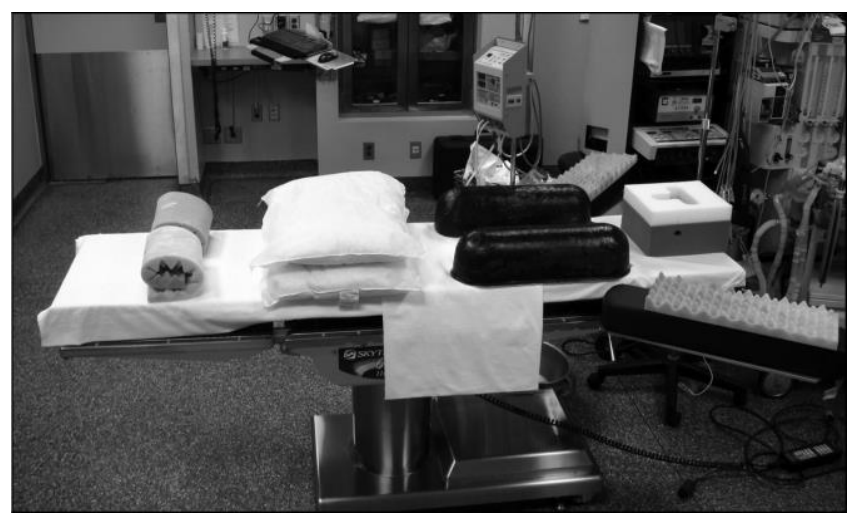




\section{Protocolo de atención en pacientes sometidos a cirugía estética}

Vol. 3, núm. 1., (2019)

José Gabriel Cayetano Lucas; Mercedes María Lucas Choez; Jaime Eduardo Jaramillo Soto; José

Fernando Calderón Ponce; Román Andrés Ortiz Burgasi; César David Pibaque Tigua

Otras posiciones utilizadas en la cirugía estética incluyen el decúbito litotómico, sentado y lateral, que se analizará en detalle más adelante en el artículo. Si bien cada posición tiene sus propios riesgos y beneficios, el enfoque descrito anteriormente para la protección de la cabeza, el cuello y las extremidades del daño al nervio y las estructuras vasculares maximiza la seguridad en cualquier posición.

\section{Protección ocular.}

La lesión ocular después de la anestesia general es poco frecuente, pero puede resultar en graves complicaciones como la discapacidad visual. (Roth, Thisted, \& Erickson, 1996) estudiaron retrospectivamente a un grupo de 60,000 pacientes durante un período de cuatro años y encontraron que la tasa de lesión ocular era aproximadamente el 0,06\%. El tipo más común de lesión es abrasión corneal, y otros incluyen conjuntivitis, lesiones químicas y ceguera.

La córnea se rasga fácilmente debido a la reducción del lagrimeo durante la anestesia. Existe un mayor riesgo de lesiones si las mascarillas se aplican incorrectamente o si las cortinas quirúrgicas se manipulan mientras los ojos permanecen abiertos. Por estas razones, los párpados deben ser cerrados con cinta antes de cualquier movimiento después de intubación. La cirugía estética de la cara limita la capacidad de proteger los ojos de esta manera. Hay poco en la literatura en cuanto a la forma más adecuada de proteger los ojos durante la cirugía facial u ocular, pero protectores de plástico de la córnea recubiertos con lubricante e insertado debajo de los párpados parece ser un método eficaz y seguro. Muchos cirujanos, sin embargo, están preocupados de que los protectores corneales distorsionan la anatomía del párpado y, por lo tanto, no lo usan durante la blefaroplastia o lifting facial. Como mínimo, se debe usar lubricante si los párpados no pueden ser tapados o protegidos de otra manera. Esta simple intervención puede ayudar a evitar la abrasión de la córnea con sutura o peor, traumatismo del globo.

En algunas posiciones, como prono, una cantidad significativa de presión se puede aplicar a los ojos. Si la presión sobre el ojo es mayor que la presión venosa, se impide la salida de sangre y la hemorragia retiniana es posible. Si la presión sobre el ojo es mayor que la presión arterial, el 


\section{Protocolo de atención en pacientes sometidos a cirugía estética}

Vol. 3, núm. 1., (2019)

José Gabriel Cayetano Lucas; Mercedes María Lucas Choez; Jaime Eduardo Jaramillo Soto; José Fernando Calderón Ponce; Román Andrés Ortiz Burgasi; César David Pibaque Tigua

flujo de sangre oxigenada se detiene y se produce la isquemia retiniana y la ceguera (Cucchiara \& Faust, 1994). Mientras que la pérdida de visión es rara, esta complicación es devastadora y debe evitarse a toda costa. Aunque no hay datos para demostrar su beneficio, muchos cirujanos plásticos actualmente usan gafas y almohadas propensas para proteger los ojos y la cara de una presión indebida en la posición prona.

\section{Condiciones Específicas.}

\section{El paciente anciano.}

La población de pacientes ancianos que buscan procedimientos cosméticos está incrementando. Comorbilidades comunes en esta población, incluyendo osteoporosis, predispone a estos pacientes a fracturas óseas incluso después de un insulto mínimo, como rodar en la operación de la mesa a la camilla (Vandam, 1993). Se requiere un mínimo de cuatro personas para trasladar a un paciente de forma segura desde la mesa de operaciones a la camilla. Cambio de posiciones mientras el paciente está intubado requiere aún más vigilancia, y atención especial a la vía aérea,

las extremidades, los genitales y los ojos son de suma importancia. Los ancianos son especialmente sensibles a hipotermia perioperatoria inadvertida. En poblaciones de alto riesgo como la anciana, la hipotermia triplica la incidencia de efectos adversos del miocardio y triplica la incidencia de infección de herida quirúrgica. Además, la importancia de la continua aspiración nasogástrica durante estos procedimientos no se puede exagerar. Aspiración que conduce a neumonía en un anciano puede ser el evento centinela que conduce a la rápida disminución en el estado del paciente durante el período postoperatorio.

\section{El paciente con obesidad mórbida.}

El Dr. J Howard Payne introdujo el término "obesidad mórbida" en la década de 1960 como un esfuerzo para convencer a las compañías de seguros y pagadores de que el tratamiento quirúrgico estaba indicado y por lo tanto reembolsable por el tratamiento de la condición de obesidad extrema. Desde entonces, la tasa de obesidad se ha disparado. Estudios del Centro Nacional de Estadísticas de Salud de los centros para el control y prevención de enfermedades muestran que 


\section{Protocolo de atención en pacientes sometidos a cirugía estética}

Vol. 3, núm. 1., (2019)

José Gabriel Cayetano Lucas; Mercedes María Lucas Choez; Jaime Eduardo Jaramillo Soto; José

Fernando Calderón Ponce; Román Andrés Ortiz Burgasi; César David Pibaque Tigua

más del $60 \%$ de los adultos estadounidenses tienen sobrepeso y el $20 \%$ son adultos con obesidad mórbida (Obesity., 2002). Como esta población ha aumentado en número, también lo ha hecho el número de pacientes con sobrepeso que buscan cirugía estética y electiva. Pacientes con obesidad mórbida plantean otro reto difícil debido a los cambios en la fisiología normal y dinámica comparada con la persona de tamaño medio. Mientras nosotros ciertamente no abogan por realizar cirugía estética electiva en la obesidad mórbida, no es infrecuente pacientes con sobrepeso que se presentan al cirujano plástico.

\section{Cirugía de contorno corporal.}

A medida que los estudios continúan mostrando el impacto beneficioso de la cirugía bariátrica sobre la salud del paciente y comorbilidades, se espera que las indicaciones para la cirugía bariátrica aumenten. Como tal, el número de procedimientos de contorno corporal probablemente aumentará también. Los pacientes de pérdida de peso masiva presentes a los cirujanos plásticos con piel y tejido subcutáneo significativamente redundantes de la piel, abdomen, muslos, nalgas, brazos, espalda y senos. Con el número de procedimientos de contorno corporal en aumento, es especialmente importante que los cirujanos presten mucha atención a la seguridad del paciente en esta población única. Una excelente reseña de todos los aspectos del cuidado del paciente con pérdida masiva de peso desde un panel de expertos se puede encontrar en (Kenkel, 2006) con consideraciones preoperatorias para pacientes con pérdida masiva de peso.

Independientemente del procedimiento de contorno a realizar, la evaluación preoperatoria de estos pacientes es de suma importancia. Una historia detallada y física en el entorno de oficina se debe resaltar las comorbilidades que puedan necesitar evaluación médica antes de la operación basado en el tipo de procedimiento bariátrico realizado, preocupaciones nutricionales incluyendo vitaminas y electrolitos. Las anomalías son relativamente comunes. Por estas razones, la evaluación temprana de los datos de laboratorio es imprescindible. Basado en las recientes recomendación de un panel de expertos, pruebas de rutina que incluyen análisis de electrolitos, hemograma completo, albúmina y prealbúmina, los niveles deben ser evaluados. Deficiencias proteicas y la anemia es común en esta población y puede dañar la curación de la herida. Se debe 


\section{Protocolo de atención en pacientes sometidos a cirugía estética}

Vol. 3, núm. 1., (2019)

José Gabriel Cayetano Lucas; Mercedes María Lucas Choez; Jaime Eduardo Jaramillo Soto; José Fernando Calderón Ponce; Román Andrés Ortiz Burgasi; César David Pibaque Tigua

hacer todo lo posible para diagnosticar y corregir desnutrición antes de la cirugía si es posible, Porque Puede ocurrir una pérdida significativa de sangre en estas cirugías prolongadas, algunas han sugerido la donación de sangre autóloga cuando surge la preocupación. Muchos pacientes tienen comorbilidades médicas que requieren asignación y evaluación médica preoperatoria. En centros con menos experiencia con pacientes con pérdida masiva de peso, la evaluación de la anestesia preoperatoria es beneficiosa.

Estos pacientes a menudo tienen una anatomía que puede complicar la intubación y muchos expertos han encontrado útil la intubación con fibra óptica (Kenkel, 2006). Además, el equipo de anestesia debe ser consciente del cambio en la anatomía de la unión gastroesofágica, aumentando la posibilidad de reflujo y aspiración. Como se discutió antes, la importancia de la aspiración nasogástrica y elevación de la cabeza siempre que sea posible no puede ser sobre enfatizado. Finalmente, los planes de acceso intravenoso se deben adaptar a la fecha programada, procedimiento y el personal de anestesia debe ser consciente de las extremidades a ser operadas. En casos donde todas las extremidades son incluidas en el campo operatorio, se puede usar una línea del cuello con seguridad.

\section{Conclusiones.}

La seguridad y la comodidad del paciente en el quirófano se han convertido crecientemente importante. Como la presión para evitar complicaciones durante los aumentos de la cirugía estética, los cirujanos plásticos deben convertirse en los principales defensores de la seguridad del paciente. Observando las directrices del Protocolo Universal tanto en el centro quirúrgico ambulatorio y hospitalario ayuda a generar un ambiente de seguridad centrado en el paciente y promueve la comunicación reflexiva entre el cirujano, personal de enfermería, el personal de anestesia y el paciente por igual. La comunicación activa permite una temprana identificación de problemas o errores futuros en la preparación preoperatoria que se puede aliviar antes del inicio de la cirugía, maximizando así la seguridad del paciente.

Posicionamiento seguro y eficaz del paciente puede evitar daño frustrante a los nervios y dolor postoperatorio muscular y articular. Además, la atención a la cabeza y el cuello durante el 


\section{Protocolo de atención en pacientes sometidos a cirugía estética}

Vol. 3, núm. 1., (2019)

José Gabriel Cayetano Lucas; Mercedes María Lucas Choez; Jaime Eduardo Jaramillo Soto; José Fernando Calderón Ponce; Román Andrés Ortiz Burgasi; César David Pibaque Tigua

procedimiento es imperativo debido al riesgo de devastación de la retina, isquemia, lesión del plexo braquial y daño a la vértebra. Protección de estructuras vitales contra daños por presión o lesión inadvertida durante un procedimiento cosmético es de suma importancia y la atención cuidadosa a la seguridad del paciente debe permanecer como objetivo número uno del cirujano plástico. Atención especial a los problemas únicos que rodean a los ancianos, los pacientes obesos y los pacientes con pérdida masiva de peso se requieren para una cirugía efectiva. Consideración de estos muchos problemas a la seguridad del paciente permite un curso perioperatorio sin problemas, por lo tanto, mejorando la experiencia de la cirugía estética mientras se optimiza la seguridad del paciente.

\section{Bibliografía.}

Abellán, J. (Mayo de 2002). Climaterio y riesgo cardiovascular. Revista de Nefrología, 105-111. www.revistenefrología.com. Obtenido de www.revist

Akinbingol, G., Borman, H., \& Maral, T. (2002). Bilateral brachial plexus palsy after a prolonged surgical procedure of reduction mammoplasty, abdominoplasty, and liposuction. . Ann Plast Surg, 219-220.

American Society of Clinical Oncology. (Enero de 2019). Síntomas de la menospausia en las mujeres. Obtenido de Cancer.Net: www.cancer.net

Arriagada, M. (2005). Recomendaciones de tratamiento durante la menospausia. Revista Chilena de Ginecología y Obstetricia, Volumen 70 N5, 340-345. www.scielo.com.cl.

Bolívar, J. (2015). Investigación Documental. México. Pax.

Bund, M., Heine, J., \& Jaeger, K. (2005). Complications due to patient positioning: Anaesthesiological considerations. Anasthesiol Intensivmed Notfallmed Schmerzther, 329-339.

Capote, M. (2011). Climaterio y Menospausia. Revista Cubana de Medicina General Integral, Volumen $27 \mathrm{~N}^{\circ} 4$, www.scielo.com.cu. 


\section{Protocolo de atención en pacientes sometidos a cirugía estética}

Vol. 3, núm. 1., (2019)

José Gabriel Cayetano Lucas; Mercedes María Lucas Choez; Jaime Eduardo Jaramillo Soto; José Fernando Calderón Ponce; Román Andrés Ortiz Burgasi; César David Pibaque Tigua

Castro, J. (2016). Técnicas Documentales. México. Limusa.

Commission., T. J. (28 de January de 2009). Universal Protocol for Preventing Wrong Site, Wrong Procedure, Wrong Person Surgery. . Obtenido de Www.jointcommission.org/PatientSafety/UniversalProtocol

Cucchiara, R., \& Faust, R. (1994). Patient positioning in Anesthesia. 1057-1073.

Davila, A. (2015). Concepto de terminos cientificos. Caracas: Oasis.

Dueñas, J. (1999). Atención a la menospausia desde el primer nivel de salud. Estadísticas Volumen 23, $N^{\circ} 6,323-389$ www.elsevier.com.

Fritzlen, T., Kremer, M., \& Biddle, C. (2003). The AANA Foundation Closed Malpractice Claims Study on nerve injuries during anesthesia care. . AANA, 347-352.

Kenkel, J. (2006). Safety considerations and avoiding complications in the massive weight loss patient. . Plast Reconstr Surg, 74S-81S.

Kohn, L., Corrigan, J., \& Donnaldson, M. (1999). To Err is Human: Building a Safer Health System. National Institute of Medicine, Committee on Quality of Health Care in America. Washington, DC.: National Academy Press.

Obesity., N. T. (2002). Medical care for obese patients: advice for health care professionals. Am Fam Physician, 81-87.

Roth, S., Thisted, R., \& Erickson, J. (1996). Eye injuries after nonocular surgery. A study of 60,965 anesthetics from 1988 to 1992. Anesthesiology, 1020-7.

Salvador, J. (Abril de 2008). Climaterio y menopausia. Obtenido de Spog: www.spog,org.pe

Santana, F. (2007). Efecto de la menopausia sobrre la prolactina de las mujeres. Scielo, Www.scielo.sid.cu. 


\section{Protocolo de atención en pacientes sometidos a cirugía estética}

Vol. 3, núm. 1., (2019)

José Gabriel Cayetano Lucas; Mercedes María Lucas Choez; Jaime Eduardo Jaramillo Soto; José Fernando Calderón Ponce; Román Andrés Ortiz Burgasi; César David Pibaque Tigua

Vandam, L. (1993). Positioning of patients for operation; Electricity, electrical safety, and instrumentation in the operating room. . Principles and Practice of Anesthesiology., 703745.

Velazco, V. (2000). Experiencias y conocimientos sobre climaterio y menospausia en mujeres de Ciudad de Mejico. Gaceta Medica de Méjico. Volumen 136 Nº, 555-564. 